\title{
Retinal vasculitis
}

\author{
M.D. Sanders and E.M. Graham
}

Medical Ophthalmology Unit, St Thomas' Hospital, London SE1 7EH, UK.

\begin{abstract}
Summary: Evidence is now accumulating on both clinical and experimental grounds that the retina is an $a$ priori source of inflammatory activity. Reactive inflammation in the retina may produce many of the clinical signs previously ascribed to uveal inflammation. Autoimmune mechanisms are probably responsible for the majority of cases of retinal vasculitis. Autoimmune retinal vasculitis occurs without other classical signs of inflammatory response in any other parts of the body. When associated systemic manifestations occur they may reflect different underlying immunopathogenic abnormalities.

Thus in diseases with predominantly arterial involvement (e.g. systemic lupus erythematosus, polyarteritis nodosa) the retinal arteries bear the brunt of this disease. In Behçet's disease the systemic involvement is usually venous and ocular involvement produces diffuse capillary and venous inflammation with areas of retinal necrosis and major vascular occlusion. The retinal appearances differ from sarcoidosis in which a granulomatous response produces characteristic periphlebitis. Finally, autoimmune retinal vasculitis produces diffuse capillary and venous damage, without any systemic signs.

In the next decade the search will be for the identification of the specific antigens initiating these disparate retinal features. Retinal $S$ antigen is a potent antigen, but rhodopsin, interphotoreceptor binding protein, and transducin all need further experimental investigation. Precise documentation will herald the dawn of new therapeutic measures based on a sound immunological fabric.
\end{abstract}

\section{Introduction}

Nowhere else in the body can a microcirculation be observed with such precision as in the retinal circulation. The vessels are distributed on the flat surface of the retina allowing accurate photographic documentation, and clear differentiation can be made between arteries, capillaries and veins. In addition, the integrity of endothelial cells can be documented by the use of fluorescein angiography, and monitored by fluorophotometry. Over the past 20 years experimental studies have shown that the retina contains several potent antigens, the most important being retinal $S$ antigen. ${ }^{1}$ An animal model of retinal vasculitis has been produced following the injection of retinal $\mathrm{S}$ antigen, and this disease produces several features in common with the posterior uveitis in humans. ${ }^{2}$ Thus retinal vasculitis may be a more accurate and preferential term reflecting inflammatory disease of the retina rather than the conventional term of uveitis. ${ }^{3}$

This review will therefore consider the most frequent causes of retinal vasculitis studied by the Medical Ophthalmology Unit at St Thomas' Hospital over the past decade. A group was formed (Figure 1) in which Dr Geraint James acted as both inspirator and catalyst. The study consists of 150 cases in which infective, ischaemic, degenerative

Correspondence: M.D. Sanders, F.R.C.P., F.R.C.S. and metastatic diseases have been actively excluded (Figure 2). Rare causes of retinal vasculitis include demyelinating disease, HLA B27 related disease and a few other eponymous conditions but the main sub-groups that will be considered in this review are:

1. Retinal arteritis

2. Retinal vasculitis in: (a) Behçet's disease; (b) sarcoidosis

3. Autoimmune retinal vasculitis

\section{Retinal arterial involvement: retinal arteritis}

Retinal arterial occlusion without detectable embolism has been noted for many years, but an increase in the number of reports has occurred over the past few years (Figure 3). An idiopathic group has emerged under a number of terms, including: retinal arteriolitis, ${ }^{4}$ retinal periarteritis, ${ }^{5}$ segmental retinal periarteritis, ${ }^{6}$ recurrent artery occlusion. ${ }^{7}$ Microangiopathy of the brain and retina ${ }^{8}$ indicates concurrent cerebral involvement, and deafness has also been associated. ${ }^{9}$ In all these reports there was major involvement of retinal arteries and arterioles but with preserved central vision and an absence of $\mathscr{O}$ inflammatory response in the eye (i.e. absence of cells in the anterior chamber or vitreous). Debate therefore exists as to whether these changes repre-

(C) The Fellowship of Postgraduate Medicine, 1988 


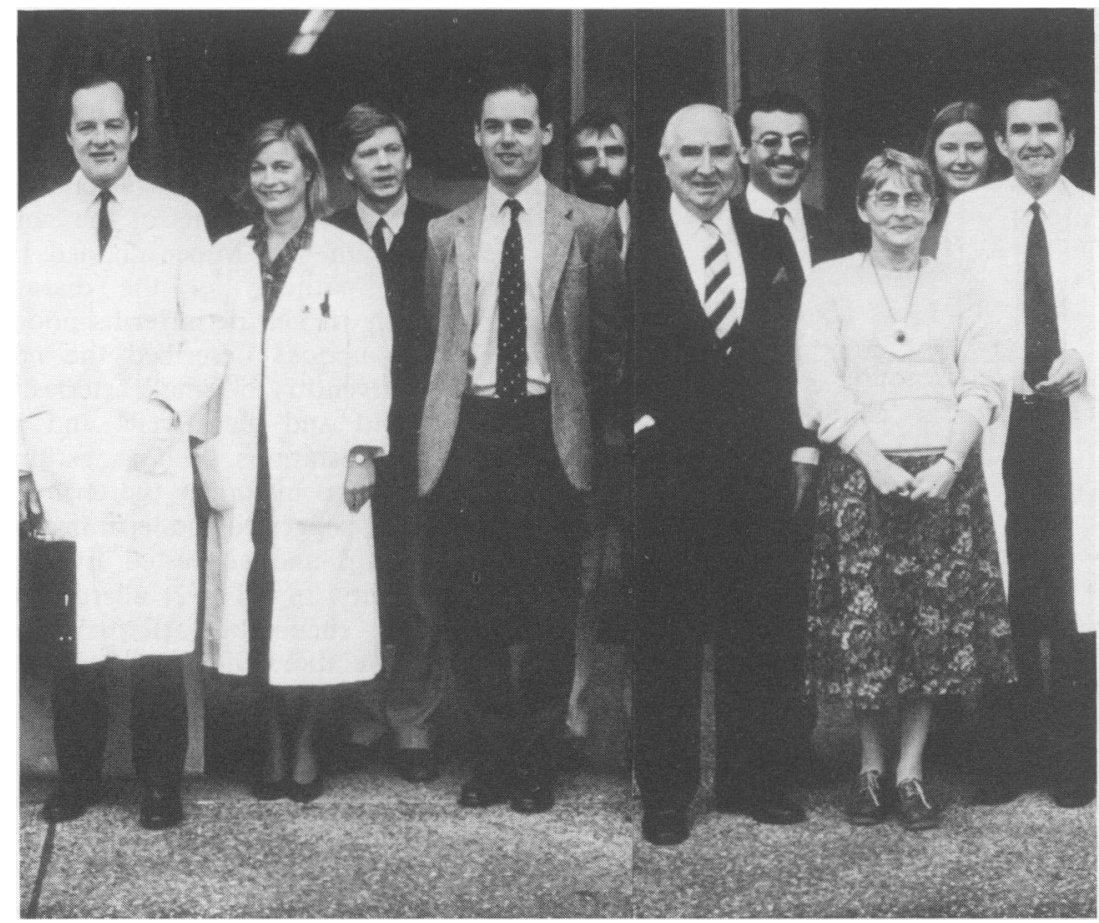

Figure 1 Retinal Vasculitis Study Group, St Thomas' Hospital 1986.

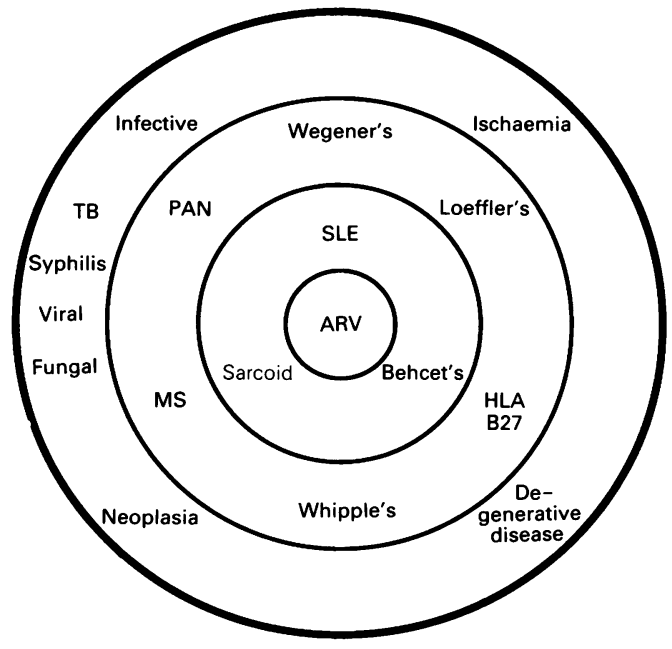

Figure 2 Diagnostic dartboard for retinal vasculitis. Outer circle represents condition that might simulate immune mediated retinal vasculitis. Second circle represents rare causes of retinal vasculitis. Inner circle - common causes of retinal vasculitis with systemic inflammatory disease. Bull's eye - autoimmune retinal vasculitis. sent immune complex deposition, an immune complex-mediated vasculitis or simply deposition of antigen in the vessel with secondary antibody reaction. Interactions between platelets and endothelial cells may be altered and recently antibodies to endothelial cells and platelets have been detected. These patients present a clinical diagnostic problem, and examples will now be given with discussion of the mechanism. In addition, arteriolar occlusions have been described in association with systemic disease of proven autoimmune nature and these will also be considered.

\section{Idiopathic retinal arteritis}

The combination of multiple retinal artery occlusions in young patients often with additional neurological involvement has been repeatedly described over the past decade. ${ }^{4,7,8}$ This syndrome has not evolved into a nosological or aetiological entity. A typical case is illustrated below:

A 40 year old cigarette smoking surgeon was admitted to the National Hospital under Dr W. Goody. He had multiple peripheral non-embolic retinal artery occlusions in both eyes, and central vision was spared. There was no evidence of inflam- 

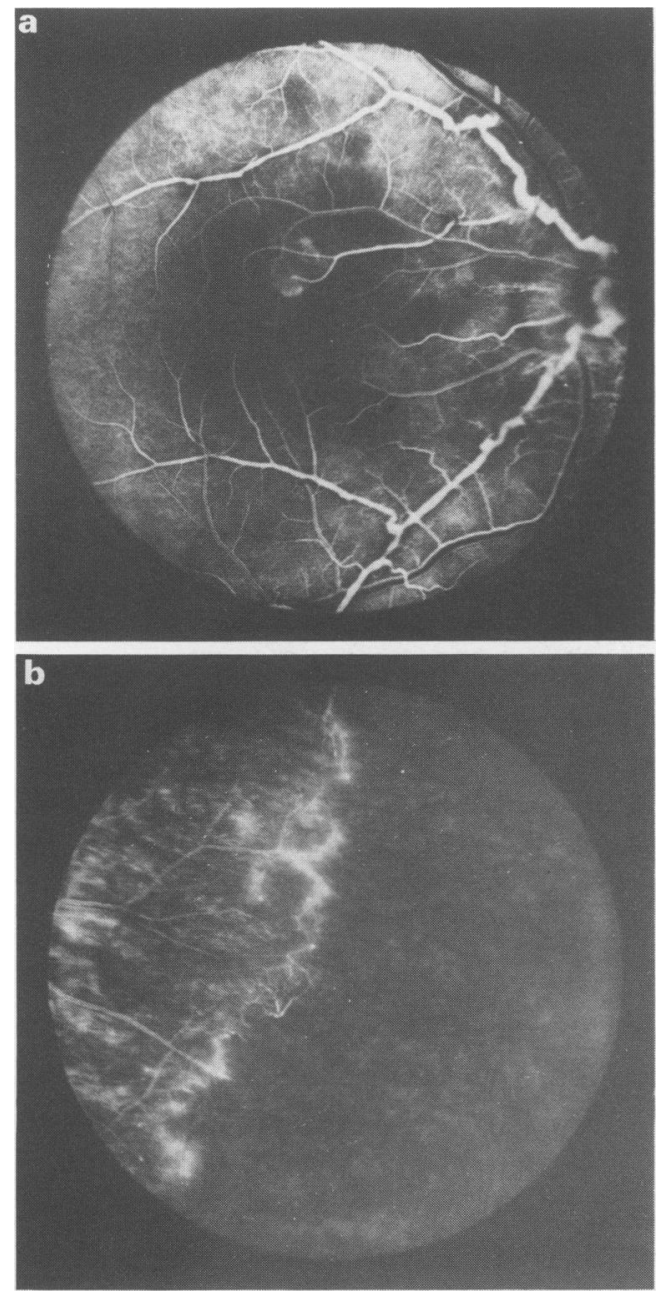

Figure 3 Retinal arteritis. (a) Multiple focal arterial abnormalities in a young man who had vision of $6 / 60$ in each eye. No systemic disease was found. (b) Peripheral nonperfusion of the retina due to ischaemia.

mation and cotton wool spots were not seen. Extensive investigations haematologically and immunologically were normal, though lipid levels were slightly increased. Macroangiography of the cerebral vessels showed no occlusive arterial disease, no angiitis and no source for embolisation in the carotid arteries. Similarly, echocardiography showed no source for cardiac embolisation.

The patient over the next year developed areas of neovascularisation at the border of non-perfused and perfused retina, which gave rise to vitreous haemorrhages. These areas of neovascularisation were treated by laser photocoagulation. No sys- temic disease evolved over 5 years of follow-up examination.

\section{Wegener's granulomatosis-polyarteritis nodosa}

It is probable that these two conditions represent different clinical spectrums of the same disease. In 1866 Kussmaul and Maier defined the gross and microscopic pathway of the disease that they appropriately named periarteritis nodosa. The term periarteritis nodosa described the nodules in the media or adventitia of small arteries. The findings of epithelioid and giant cells and propensity to allergic manifestations such as asthma led Churg and Strauss ${ }^{10}$ to introduce the term allergic granuloma. Zeek ${ }^{11}$ suggested the term necrotising angiitis for this group and suggested five subgroups: (1) hypersensitivity angiitis; (2) allergic granulomatous angiitis; (3) rheumatic arteritis; (4) periarteritis nodosa; (5) temporal arteritis.

Wegener in 1936 defined a disease characterized by necrotising granulomatous vasculitis of the upper and lower respiratory tract progressing to renal involvement with glomerulonephritis. This condition has been further subdivided in several different subgroups, one of which has granulomatous features, e.g. sarcoidal Wegener's, and another with lymphoreticular features, e.g. lymphomatoid granulomatosis. ${ }^{12}$

The proximity of the orbit to the upper nasal tract may account for the high incidence of ocular involvement which occurred in $47 \%$ of the patients described by Haynes et al., ${ }^{13}$ with orbital involvement being most common, and only one patient having optic disc vasculitis. A review of eight patients with ocular involvement at St Thomas' Hospital showed retinal involvement in only one case and this case represented the rare group of sarcoidal Wegener's. The patient was a 12 year old boy presenting with transverse myelitis, pneumonia and uveitis. The retinal veins showed perivasculitis in the periphery and there was no arterial involvement. ${ }^{14}$ The retinal involvement in this case resembles the retinopathy of sarcoidosis and this is consistent with the histological similarity to sarcoidosis. Ocular involvement in periarteritis nodosa is extremely rare and our experience extends to only two cases which are included in this group. In one patient bilateral sixth nerve palsy was accompanied by branch arteriolar occlusions. In the other patient a central vein occlusion was the only ocular manifestation.

\section{Churg-Strauss syndrome}

Allergic angiitis and granulomatosis forms part of the spectrum of disseminated systemic necrotising 
vasculitis and pathological examination shows fibrinoid necrosis of small and medium sized arteries. Churg-Strauss differs from polyarteritis nodosa in the degree of inflammatory involvement of small vessels including capillaries and veins. There are several reports of ocular involvement in this syndrome, and retinal arterial occlusions have been a feature. ${ }^{15,16}$ It is interesting that there is a marked peripheral and tissue eosinophilia in the ChurgStrauss syndrome which is also a feature of the hypereosinophilic syndrome.

\section{Loeffler's eosinophilic syndrome}

An interesting young patient with endocarditis, eosinophilia and renal disease was referred by $\mathrm{Dr}$ Guy Neild. Fundus examination showed numerous retinal arterial occlusions in both eyes without signs of retinal infarction. Fluorescein angiography confirmed arteriolar occlusion, and there was no evidence of choroidal involvement. Autopsy was performed which showed occlusion of arterioles by fibrinoid material but without perivascular inflammation. The patient was diagnosed as Loeffler's fibroblastic endocarditis which represents one end of the spectrum of the hypereosinophilic syndrome. ${ }^{17}$

\section{Systemic lupus erythematosus}

Systemic lupus erythematosus (SLE) is a wellestablished non-organ-specific autoimmune disease and both human and animal studies suggest that genetic, endocrine and environmental factors are involved.

Retinal involvement occurs in 5 to $10 \%$ of cases and this association provides strong evidence to support the concept of autoimmune disease producing a retinopathy. SLE produces multisystem involvement and definitive diagnostic criteria are available with the detection of extractable antinuclear antibodies, and in particular anti-DNA antibodies.

The retinal features are due to arterial occlusion and the characteristic findings are cotton wool spots (Figure 4), larger retinal infarcts and optic disc infarction. Embolic occlusion from cardiac vegetations may occur, but the retinal changes are usually manifest without clinical or post-mortem changes of Libman-Sachs endocarditis, and without visible calcific retinal emboli.

A particular feature of the retinopathy of SLE is that the veins are not involved, and there are no inflammatory changes in the anterior chamber or vitreous. Pathological examination shows occlusion of arterioles by fibrinoid material without vasculitis, though vasculitis or immunoglobulin deposition
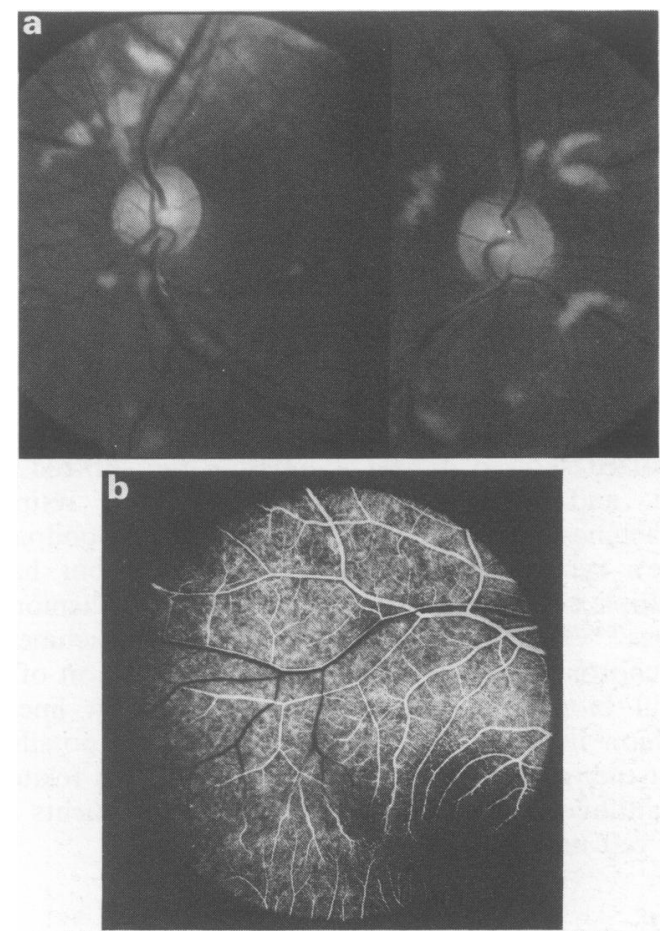

Figure 4 Systemic lupus erythematosus. (a) Multiple cotton wool spots. (b) Attenuated arterioles with reduced retinal perfusion.

may be found in other vessels. In cerebral SLE microvascular damage is a characteristic neuropathological finding and speculation suggests two immunopathogenic mechanisms. Firstly, immune complex-mediated vascular injury and secondly, direct autoantibody-mediated attack on the vascular endothelium. The cross reactivity of anti-cardiolipid antibodies with cell membrane phospholipids may suggest an explanation for the endothelial cell damage, as high levels of anticardiolipid antibodies are a feature of some cases of SLE. ${ }^{18}$

A third and particularly interesting aspect is the finding of antibodies to neuronal membrane antigens, which may be shared with lymphocyte antigens. In one patient in this series pathological examination of the eyes was obtained of a young boy with cerebral and ocular SLE. Fibrinoid occlusion of retinal arteries occurred without arteritis, but extensive cellular infiltration and vasculitis occurred in choroidal vessels. The choroidal inflammatory signs may reflect the increased permeability of the choroid vessels, as a result of immune complex disease involvement, and cerebral arteries showed occlusion though an arteritis was found in the meningeal vessels. ${ }^{14}$ 


\section{Goodpasture's syndrome}

Goodpasture in 1919 described a patient with haemoptysis, anaemia and proteinuria, and postmortem examination showed pulmonary alveolar haemorrhages and glomerulonephritis. Immunological studies have shown the deposition of immunoglobulin $\mathrm{G}$ (IgG) in a linear pattern in basement membrane of the kidney and lung. Similar changes were reported in the eye by Jampol et al. ${ }^{20}$ with linear deposition of IgG in Bruchs membrane and the basement membranes of the choroidal vessels. Clinical examination of the two patients he reported showed ischaemic areas in the choroid in one, and both had non-rhegmatogenous retinal detachments. We have seen one case of Goodpasture's syndrome who was normotensive but had multiple cotton wool spots and occasional haemorrhages. ${ }^{21}$ There were no signs of retinal detachment or choroidal disease. Histological examination of a renal biopsy showed IgG deposition in a linear fashion in the basement membrane. It is possible that the retinopathy in this condition also is related to antibodies to endothelial cells or constituents of the cell membrane.

\section{Pathogenesis of retinal arteritis}

A group of conditions therefore exists presenting in normotensive patients without a source for embolisation in which retinal and cerebral infarction occurs. The conditions include SLE, polyarteritis nodosa, Churg-Strauss syndrome, Goodpasture's syndrome and Loeffler's eosinophilic syndrome, with an additional idiopathic group. The clinical features of this group are: (1) cotton wool spots indicating precapillary arteriolar involvement; (2) retinal branch arteriolar occlusions (a) with infarction, (b) without signs of infarction, suggesting that patients were not examined during the acute phase or that evolution of occlusion was chronic in nature; (3) no signs of ocular inflammation.

It is interesting to speculate that this group may exhibit antibodies or autoantibodies against endothelial cells or constituents of their cell membranes with immune complex deposition. It is established that retinal and cerebral endothelial cells have similar anatomical and physiological characteristics and this may extend to their immunological properties. In addition, the finding of antineuronal antibodies in cerebral SLE raises the question of whether shared antigens occur in the retina and brain. The assumed autoimmune nature of the associated disease lends credence to the view that this is an immunological process and though a true vasculitis has not yet been recorded on histological examination the mechanism is presumably inflammatory.

\section{Retinal vasculitis}

Retinal vasculitis is defined as inflammation of the eye (cells in the anterior chamber and/or vitreous) in association with retinal vascular disease; this may be ophthalmoscopically evident or demonstrable only by fluorescein angiography. Classically the inflammation involves the retinal veins and post- $\vec{\circ}$ capillary vessels but rarely the arterioles. Retinal vasculitis occurs in three clinical situations either as an isolated clinical manifestation or in association with either sarcoidosis or Behçet's disease. The aetiology is unknown but autoimmune processes are deemed responsible even when retinal vasculitis occurs without other systemic manifestations.

Autoimmunity represents an immune response directed against the host which may become a $\stackrel{+}{+}$ pathological factor if it exceeds a certain threshold. Certain criteria are necessary for establishing an autoimmune disease:

1. Autoreactive antibodies or lymphocytes can be detected.

2. The autoimmune reaction can be shown to $\overrightarrow{\mathscr{O}}$ damage target cells directly or by secondary imm通 nological and inflammatory reactions.

3. The disease can be transmitted auto-antibodies.

4. Immunomanipulation ameliorates the disease.

Many of these criteria are established in retinal vasculitis and in addition we have developed an animal model of the disease which closely simulates the disease in man on immunological, clinical and pathological grounds.

\section{The clinical features of retinal vasculitis with} systemic disease

\section{Sarcoidosis}

Sarcoidosis is a multisystemic granulomatous dis- 욱 order of unknown aetiology with pulmonary, cutaneous and ocular manifestations. Ocular involvement occurs in approximately $25 \%$ of $\bar{N}$ cases. ${ }^{22,23}$ The ophthalmologist by detecting ocular o involvement may aid the diagnosis of a multisystem 0 disease, but furthermore the retinal findings may be $\underset{\omega}{N}$ pathognomonic. ${ }^{24}$ In the present series there were ${ }^{\sigma}$ 17 biopsy proven cases of sarcoidosis. In this group 0 in addition to pulmonary and ocular involvement the following systems were involved: arthritis $7, \stackrel{\infty}{\rightarrow}$ cutaneous 2, central nervous system 3 , Kveim + ve 10 and biopsy lung/skin 7 . 
Retinal involvement was characterized by focal periphlebitis and this is a characteristic feature of sarcoidosis (Figure 5). Predominant changes occur around the veins and arterial involvement is virtually never seen. Clinical and fluorescein angiographic features include: focal periphlebitis $(78 \%)$, diffuse capillary leakage $(44 \%)$, new vessel formation $(5 \%)$ and acute retinopathy.

Iritis occurred in $41 \%$ of cases and retinal and optic disc involvement may occur without involvement of the anterior chamber. Retinal vein occlusion and infiltration of the retina are not seen in sarcoidosis and serve to differentiate sarcoidosis from Behçet's disease. There were no cases of the acute retinopathy of sarcoidosis in this series though this rare presentation has been seen in two other cases and included in other reports. ${ }^{24,25}$ The acute retinopathy usually progresses to capillary closure and neovascularisation.

\section{Behçet's syndrome}

Behçet's syndrome is a multisystem disease affecting young adults with a classical triad of mouth ulcers, genital ulcers and iritis. Recognized in the writing of Hippocrates but eventually named after a Turkish dermatologist, Professor Halusi Behçet, this condition provides a major challenge to ophthalmology because of the ocular devastation it produces. Clinically patients give a long history of mouth ulcers, usually from childhood, and often with up to 12 ulcers occurring simultaneously. Ocular

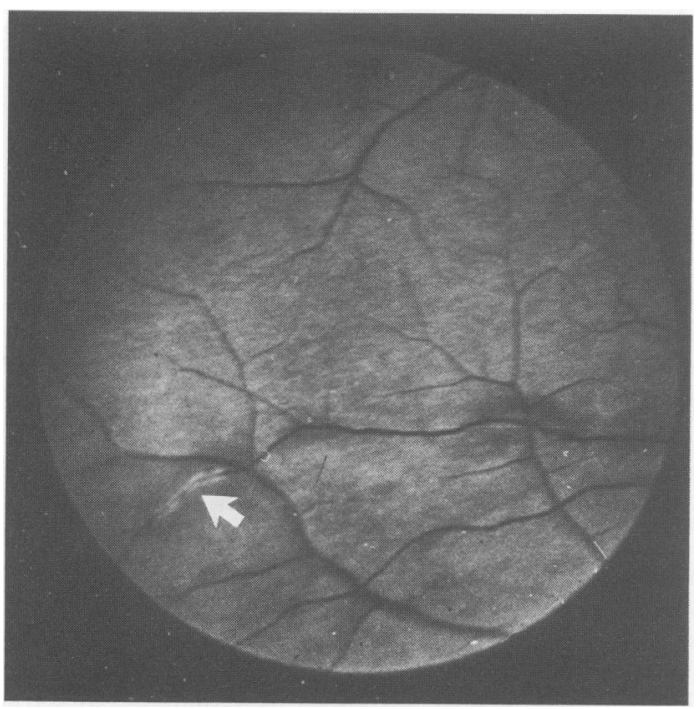

Figure 5 Sarcoidosis. Peripheral retinal periphlebitis, a pathognomonic feature of retinal sarcoidosis. features present in the second and third decades and in addition to ocular features there are mucocutaneous, intestinal, vascular, urological and neurological manifestations. Death from Behçet's syndrome is rare (3 to $4 \%$ ) and the disease usually burns itself out, leaving a blind or partially blind patient. The underlying histopathological lesion in all organ systems is vasculitis. This includes perivascular infiltrates of monocular cells, swelling and proliferation of endothelial cells leading to partial obliteration of small vessels and fibrinoid degeneration. ${ }^{26}$ Despite a wide variety of immunological abnormalities, there is no specific diagnostic test and the clinician rather than the immunologist holds the key to diagnosis. The high incidence of the haplotype HLA B5 has been described in all epidemiological studies of ocular Behçet's, including those in both Japan and Europe. HLA B27 however may be found in the arthritis type of Behçet's syndrome and HLA B12 in the mucocutaneous type. ${ }^{27}$ There may be a disequilibrium linkage between HLA B5 and HLA A2 in the ocular group with retinal vein occlusion. ${ }^{28}$ Immune complexes are found in patients and in exacerbations of disease an increase in IgG complexes. ${ }^{27}$

Ocular involvement has certain specific and diagnostic features, and occurs in $80 \%$ of patients. Behçet's syndrome produces the most aggressive pattern of vasculitis and in the last decade vision was deemed to be lost within 3.36 years after onset of ocular involvement. ${ }^{29}$ However, the prognosis has been improved by early recognition and aggressive treatment by steroids, immunosuppressives and cyclosporins. Excellent reviews are available ${ }^{26,30}$ so that this discussion will concentrate on the retinal features.

Emphasis must first be placed on the fact that this is a retinal and not a choroidal disease, on both clinical and pathological evidence. Hypopyon is a rare feature occurring in our series in $13 \%$ of cases, and iritis (48\%) is mild and does not usually produce visual loss. Retinal involvement has characteristic features first indicated in $1979^{28}$ but supported by the present series. Our present series comprises 39 patients and $65 \%$ were under the age of 40 years. The general features include: mouth ulcers $100 \%$, genital ulcers $66 \%$, skin lesions $51 \%$, arthritis $46 \%$, thrombophlebitis $43 \%$ and central nervous system involvement.

The ocular features could be subdivided into four distinct groups: diffuse capillary leakage $100 \%$, branch vein occlusion $62 \%$, infiltrations $40 \%$, and terminal stage with optic atrophy and macular degeneration. Diffuse capillary leakage occurred in all cases, as observed by fluorescein angiography and affected the posterior pole (Figure 6). Maximal involvement occurred at the disc, the arcuate capil- 

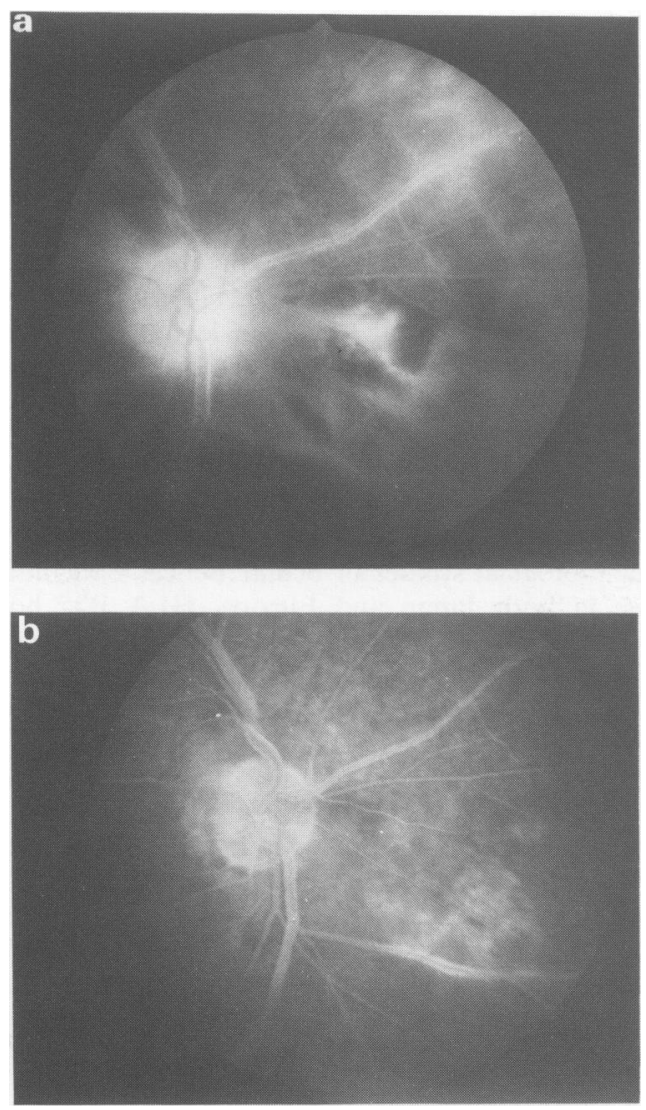

Figure 6 Behçet's disease. (a) Severe retinal vascular leakage on fluorescein angiography of this left eye with focal infiltration. (b) One month after prednisolone treatment there is marked resolution of the leakage of dye.

laries and the macular capillaries. A peripheral periphlebitis or focal periphlebitis was not seen. Extensive areas of retinal non-perfusion were not seen without venous occlusion, and neither was neovascularisation a feature. These factors serve to distinguish the retinopathy of Behçet's syndrome from other types of vasculitis.

Branch vein occlusion occurred in $62 \%$ of cases and could involve small macular vessels or hemisphere vein occlusion. Central retinal vein occlusion was not seen. Factors contributing to the pathogenesis include inflammation of the vessel wall but in addition elevated blood viscosity was found in two patients. Branch vein occlusion, particularly when the macular vessels are involved, provides a major source of the visual disability associated with Behçet's syndrome.
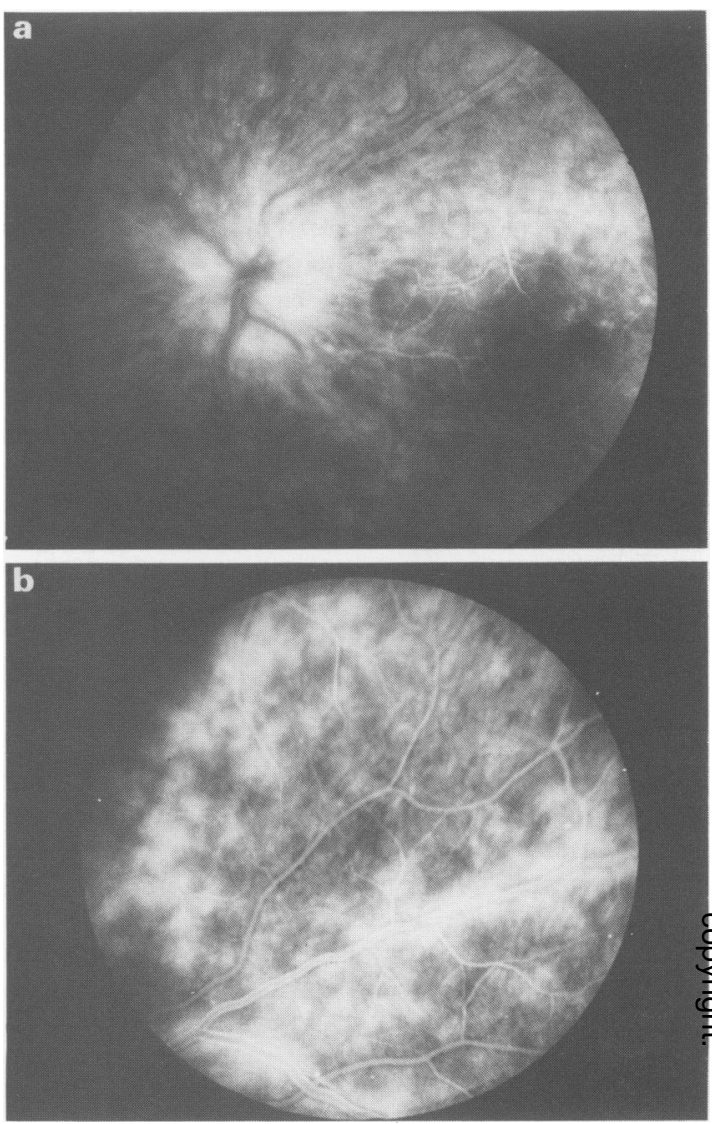

Figure 7 Autoimmune retinal vasculitis. Severe bilateral visual loss was seen in this 18 year old girl. There was disc oedema on the left with diffuse capillary leakage on fluorescein angiography (a), extension into the periphery with marked perivenous leakage (b).

Retinal infiltrations present deep pale areas, often surrounded by haemorrhages. They resolve promptly on steroid treatment suggesting they are manifestations of inflammation rather than ischaemia and may be seen as a retinal manifestation of the hypopyon.

Retinal infiltrations and branch vein occlusions are specific and pathognomonic features of the retinopathy of Behçet's disease and are not seen in any other condition. Despite modern therapeutic measures and the use of cyclosporin in our patients $^{31}$ the visual prognosis was bad: $46 \%-6 / 18$ in both eyes, $80 \%-6 / 18$ in one eye. Immunological tests were not of diagnostic value, though HLA B5 occurred in $65 \%$ of cases and in $27 \%$ of cases the combination HLA A2 and B5 was associated with branch vein occlusions. 


\section{The clinical features of autoimmune retinal vasculitis}

Sixty-seven patients had retinal inflammatory disease without other systemic features. The finding of autoimmune features and the establishment of an animal model justifies the appellation for this group of 'autoimmune retinal vasculitis' (ARV).

There were 67 patients in this group with a female predominance ( 27 males, 40 females). The majority $(72 \%)$ of patients were under 40 years of age: under age $20-21 \%, 20-29-21 \%$, and $30-39$ $-30 \%, 15 \%$ were $40-49$ years of age and $13 \%$ over 50. Examination showed no evidence of cutaneous, neurological or pulmonary disease and specific tests were negative. Immunological tests were similar to those patients with retinal vasculitis and a systemic disease, though the incidence of retinal autoanti-

\section{References}

1. Wacker, W. \& Lipton, M. Experimental allergic uveitis. Homologous retina as uveitogenic antigen. Nature 1965, 206: 253.

2. Stanford, M.R., Graham, E.M., Kasp, E., Brown, E.L., Dumonde, D.C. \& Sanders, M.D. Retinal vasculitis: correlation of animal and human disease. Eye 1987, 1: 69-77.

3. Sanders, M.D. Retinal arteritis, retinal vasculitis and autoimmune retinal vasculitis. Eye 1987, 1: 441-465.

4. Jampol, L.M., Isenberg, S.J. \& Goldberg, M.F. Occlusive retinal arteriolitis with neovascularization. Am J Ophthalmol 1976, 81: 583-589.

5. Griffin, A.O. \& Bodian, M. Segmental retinal periarteritis. Am J Ophthalmol 1959, 47: 544.

6. Orzalesi, N. \& Riccardi, L. Segmental retinal periarteritis. Am J Ophthalmol 1971, 72: 55-59.

7. Gass, J.D.M., Tiedeman, J. and Thomas, M.A. Idiopathic recurrent branch retinal artery occlusion. Ophthalmology 1986, 93: 1148-1157.

8. Susac, J.O., Hardman, J.M. \& Selhorst, J.B. Microangiopathy of the brain and retina. Neurology 1979 , 29: 313-316.

9. Delaney, W.V. \& Torrisi, P.F. Occlusive retinal disease and deafness. Am $J$ Ophthalmol 1976, 82: 232-236.

10. Churg, J. \& Strauss, L. Allergic granulomatosis, allergic angiitis and periarteritis nodosa. Am J Pathol 1951, 27: 227-302.

11. Zeek, P.M. Periarteritis nodosa, a critical review. Am $J$ Clin Pathol 1952, 22: 777-790.

12. Liebow, A.A. Pulmonary angiitis and granulomatosis. Am J Rev Resp Dis 1975, 108: 1-18.

13. Haynes, B.F., Fishman, M.L., Fauci, A.S. and Wolf, S.M. The ocular manifestations of Wegener's granulomatosis. Am J Med 1977, 63: 131-141.

14. Spalton, D.J., Graham, E.M., Page, N.G.R. \& Sanders, M.D. Ocular changes in limited forms of Wegener granulomatosis. Br J Ophthalmol 1981, 65: $553-563$. bodies (measured by indirect immunofluorescence) was lower in those with systemic disease only.

Two subgroups could be demonstrated, one with positive retinal antibodies (34 patients) and the other with negative retinal antibodies (36 patients). There were no clinical differences between these groups and one may speculate that in the negative group tests may have been directed at the wrong epitope of retinal $S$ antigen, or indeed the wrong antigen.

There are two characteristic retinal features of autoimmune retinal vasculitis (Figure 7): diffuse capillary leakage $(83 \%)$ and capillary closure and new vessels $(23 \%)$. In particular there were no infiltrations or branch vein occlusions in contrast to Behçet's disease, no evidence of peripheral periphlebitis as seen in sarcoidosis, and no signs of arterial occlusion.

15. Dagi, L.R. \& Currie, J. Branch retinal artery occlusion in the Churg-Strauss Syndrome. J Clin Neuro Ophthalmol 1985, 5: 229-237.

16. Weinstein, J.M., Chui, H. \& Lane, S. Churg-Strauss syndrome (allergic granulomatous angitis). Arch Ophthalmol 1983, 101: 1217-1220.

17. Chusid, M.J., Dale, D.C., West, B.C. \& Wolf, S.M. The hypereosinophilic syndrome. Medicine 1925, 54: 1.

18. Hughes, G.R.V. Thrombosis, abortion, cerebral disease and the lupus anticoagulant. $\mathrm{Br} M e d J$ 1983, 287: 1088-1089.

19. Graham, E.M., Spalton, D.J., Barnard, R.O., Garner, A. \& Ross Russell, R.W. Cerebral and retinal changes in systemic lupus erythematosus. Ophthalmology 1985, 92: $444-448$.

20. Jampol, L.M., Lahar, M. \& Albert, D.M. Ocular clinical findings and basement membrane changes in Goodpasture's syndrome. 1975, 79: 452.

21. Sanders, M.D., Retinal vasculitis, a review. $J \boldsymbol{R}$ Soc Med 1979, 908-915.

22. Siltzbach, L.E., James, D.G., Neville, E. et al. Course and prognosis of sarcoidosis around the world. Am J Med 1985, 57: 847.

23. Jabs, D.A. \& Johns, C.J. Ocular involvement in chronic sarcoidosis. Am J Ophthalmol 1986, 102: 297-301.

24. Sanders, M.D. \& Shilling, J.S. Retinal choroidal and optic disc involvement in sarcoidosis. Trans Ophthalmol Soc UK 1976, XCVI: 140-144.

25. Spalton, D.J. \& Sanders, M.D. Fundus changes in histologically confirmed sarcoidosis. $\mathrm{Br} J$ Ophthalmol 1981, 65: 348-358.

26. Michelson, J.B. \& Chisari, F.V. Behçet's disease. Surv Ophthalmol 1982, 26: 190-203.

27. Lehner, T. \& Batchelor, Jr. In: Lehner, T. \& Barnes, C.G. (eds) Classification and Immunogenetic Basis of Behçet's Syndrome. Academic Press, London, 1979. 
28. Sanders, M.D. Ophthalmic features of Behçet's disease. In: Lehner, T. \& Barnes, C.G. (eds) Behçet's Syndrome. Academic Press, London, 1979, pp 183-189.

29. Mamo, J.G. The rate of visual loss in Behçet's disease. Arch Ophthalmol 1970, 84: 451-452.

30. Shimizu, T. \& Saito, Y. Behçet's syndrome. Clin Sci 1971, 17: 451-453.
31. Binder, A.L., Graham, E.M., Sanders, M.D., Dinning, W., James D.G. \& Denman, A.M. Cyclosporin A in the treatment of severe Behçet's uveitis. $B r J$ Rheumatol 1987, 26: 285-291.

32. Stanford, M.R., Brown, E.C., Kasp, E., Graham, E., Sanders, M.D. \& Dumonde, D.C. Experimental posterior uveitis I. A clinical, angiographic and pathological study. Br J Ophthalmol 1987, 71: 585-592. 